\title{
BMJ Open Surgical candidacy and treatment initiation among women with cervical cancer at public referral hospitals in Kampala, Uganda: a descriptive cohort study
}

\author{
Megan Swanson (D) , ${ }^{1}$ Miriam Nakalembe, ${ }^{2}$ Lee-may Chen, ${ }^{1}$ Stefanie Ueda, ${ }^{1}$ \\ Jane Namugga, ${ }^{3}$ Carol Nakisige, ${ }^{4}$ Megan J Huchko ${ }^{5,6}$
}

To cite: Swanson M, Nakalembe M, Chen L, et al. Surgical candidacy and treatment initiation among women with cervical cancer at public referral hospitals in Kampala, Uganda: a descriptive cohort study. BMJ Open 2020;10:e039946. doi:10.1136/ bmjopen-2020-039946

- Prepublication history for this paper is available online. To view these files, please visit the journal online (http://dx.doi. org/10.1136/bmjopen-2020039946).

Received 07 May 2020

Revised 23 September 2020

Accepted 26 October 2020
Check for updates

(C) Author(s) (or their employer(s)) 2020. Re-use permitted under CC BY-NC. No commercial re-use. See rights and permissions. Published by BMJ.

For numbered affiliations see end of article.

Correspondence to Dr Megan Swanson; megan.swanson@ucsf.edu

\section{ABSTRACT}

Objectives This study aimed to report the proportion of women with a new diagnosis of cervical cancer recommended for curative hysterectomy as well as associated factors. We also report recommended treatments by stage and patterns of treatment initiation. Design This was an observational cohort study. Inperson surveys were followed by a phone call.

Setting Participants were recruited at the two public tertiary care referral hospitals in Kampala, Uganda. Participants Adult women with a new diagnosis of cervical cancer were eligible: 332 were invited to participate, 268 met the criteria and enrolled, and 255 completed both surveys.

Primary and secondary outcomes measures The primary outcome of interest was surgical candidacy; a secondary outcome was treatment initiation. Descriptive and multivariate statistical analyses examined the associations between predictors and outcomes. Sensitivity analyses were performed to examine outcomes in subgroups, including stage and availability of radiation.

Results Among 268 participants, $76 \%$ were diagnosed at an advanced stage (IIB-IVB). In total, $12 \%$ were recommended for hysterectomy. In adjusted analysis, living within $15 \mathrm{~km}$ of Kampala (OR 3.10, 95\% Cl 1.20 to 8.03 ) and prior screening (OR $2.89,95 \% \mathrm{Cl} 1.22$ to 6.83 ) were significantly associated with surgical candidacy. Radiotherapy availability was not significantly associated with treatment recommendations for early-stage disease (IA-IIA), but was associated with recommended treatment modality (chemoradiation vs primary chemotherapy) for locally advanced stage (IIB-IIIB). Most (67\%) had started treatment. No demographic or health factor, treatment recommendation, or radiation availability was associated with treatment initiation. Among those recommended for hysterectomy, $55 \%$ underwent surgery. Among those who had initiated treatment, $82 \%$ started the modality that was recommended.

Conclusion Women presented to public referral centres in Kampala with mostly advanced-stage cervical cancer and few were recommended for surgery. Most were able to initiate treatment. Lack of access to radiation did not
Strengths and limitations of this study

- The study was conducted at the two publicly funded national referral hospitals providing subsidised specialty cancer care to Uganda's population of approximately 42 million people.

- This is the first study in the region to report treatment recommendations, including proportion of those assessed to be surgical candidates, at the time of diagnosis of cervical cancer, the most common cancer among women in Uganda.

- Because this is a convenience sample of women at tertiary care centres in the urban capital city, the study likely overestimates the proportion of surgical candidates among all incident cases of cervical cancer nationwide.

During the first half of the study period, there was no available incountry radiotherapy, while in the second half the new radiation machine was commissioned and radiotherapy was again available, allowing us to explore the association between availability of radiation and treatment recommendations by stage, although the study was underpowered to rigorously assess the impact.

- This is one of the first studies in the region to report on treatment initiation and the first to investigate the association of treatment recommendations, availability of radiation as well as demographic and clinical factors on treatment initiation among women with cervical cancer.

significantly increase the proportion of early-stage cancers recommended for hysterectomy.

\section{INTRODUCTION}

Cervical cancer is the fourth most common cancer among women worldwide: 570000 new cases and 311000 deaths estimated in 2018. ${ }^{1}$ Cervical cancer is over-represented in low-income and middle-income countries (LMICs); the highest incidence and 
mortality rates are in Southern and Eastern Africa. In Uganda, cervical cancer is the most common malignancy and responsible for the greatest cancer-related mortality among women. ${ }^{2}$ While population-based screening has dramatically reduced incidence in high-income countries, incidence and mortality have risen in LMICs like Uganda, ${ }^{3}$ where less than $5 \%$ of women have ever been screened. ${ }^{4}$

In Uganda, most women with cervical cancer are diagnosed at an advanced stage, ${ }^{5}$ although poor record keeping affects the accuracy of estimates. The Kampala Cancer Registry lacked stage data for half of the 261 cervical cancer cases reported in a 3-year period; $73 \%$ of the remaining cases were stage II $+{ }^{6}$ As a result, 5 -year overall survival for all cervical cancer cases is approximately $18 \%{ }^{7}$ Cervical cancer, especially early stage, is potentially curable with surgery and/or radiation with or without chemotherapy, but multisystem obstacles often block access to treatment. ${ }^{8}$

Challenges to accessing surgery include limited specialty surgical training, ${ }^{910}$ few anaesthesia providers ${ }^{11}{ }^{12}$ and a lack of sufficient banked blood. ${ }^{13}$ In response to the lack of trained providers, the Uganda Cancer Institute (UCI) and the Mulago National Referral Hospital (MNRH), with support from the African Development Bank and external mentorship from gynaecological oncologists in the USA, have created a fellowship programme in gynaecological oncology. The programme has just graduated the first two locally trained Ugandan gynaecological oncologists in 2019.

Access to radiation, the gold standard treatment for bulky early-stage disease and locoregionally advanced cervical cancer, is limited in sub-Saharan Africa ${ }^{14}$; Uganda is no exception. ${ }^{15}$ After the single functioning radiation machine was determined broken beyond repair in March 2016, Uganda was without radiotherapy until the new cobalt machine was inaugurated in January 2018. During this time, a private hospital in Nairobi, Kenya worked with the Ugandan Ministry of Health to offer subsidised radiation to select patients (no specific number of spots were designated for cervical cancer), although out-ofpocket expenses would have been prohibitive for most Ugandans. ${ }^{16}{ }^{17}$ It is unclear how many patients ultimately benefited. Chemotherapy is provided free of charge at government facilities, but shortages are frequent. ${ }^{18}$

Most care for cervical cancer in Uganda is provided at two specialty public hospitals: MNRH and UCI. Clinical consultations are free of charge; there are nominal fees associated with radiotherapy and surgery. In response to the challenges obtaining radiotherapy in 2016-2018, providers at UCI and MNRH developed a protocol for treating locally and regionally advanced cervical cancer with neoadjuvant chemotherapy followed by possible radical hysterectomy, an evidence-based strategy supported by the American Society of Clinical Oncology (ASCO) Resource-Stratified Treatment Guidelines. ${ }^{19-22}$

The ASCO Resource-Stratified Treatment Guidelines also suggest a broader role for hysterectomy for cervical cancer in settings without access to radiation. While radical hysterectomy is a theoretical consideration for stage IA2-IIA2, in maximally resourced settings, chemoradiation is the preferred treatment modality for women with stage IB2-IIA2 disease given equivalent outcomes with less morbidity. ${ }^{23}$ In settings without radiotherapy, primary treatment with hysterectomy may be considered as an alternative for women with stage IB2-IIA2 disease. ${ }^{19}$

We sought to describe the presentation of cervical cancer at MNRH and UCI in Kampala, Uganda, specifically the proportion of patients recommended for hysterectomy (simple or radical) at the time of diagnosis and the factors associated with surgical candidacy. We also sought to describe primary treatment recommendations, utilisation patterns and factors associated with successful treatment uptake, in the context of variably available treatments.

\section{METHODS}

\section{Participants and study setting}

From April 2017 through September 2018, we surveyed women over 18 years old presenting to care at either of two government-sponsored referral hospitals (MNRH and UCI) with a new diagnosis of cervical cancer and invited participation in baseline and follow-up surveys. This analysis is part of a larger study examining patterns of delay in accessing treatment.

Research assistants sequentially approached women in clinics and invited them to participate. Interested participants were screened for eligibility. Women $>18$ years old able to understand English or Luganda with a histopathological diagnosis of primary cervical cancer and a clinical stage assigned by a gynaecologist in the gynaecological oncology division at MNRH or UCI were eligible to participate. Participation was voluntary and participants were reimbursed in part for travel expenses (20000 Ugandan shillings or approximately US $\$ 5$, the standard amount approved by the local ethics committees who reviewed the study). Individual informed consent was obtained.

\section{Data collection}

The first part of the survey was administered in the clinic. Research assistants verbally collected demographic and basic health information, including a reproductive health history from participants before collecting information about each individual's journey to care. This quantitative survey was adapted from a validated survey instrument to measure time intervals and factors correlated with delay in accessing breast cancer care. ${ }^{24}$ Minor modifications were made in order to map the survey onto the model of pathways to treatment, a theoretical model adapted from the Andersen model for understanding and describing the process of obtaining diagnosis and treatment for cancer, ${ }^{25-27}$ and to make the questions specific to cervical cancer. Participants' medical records were used to corroborate information on histology, stage and grade of cervical tumours, as well as the dates of the biopsy, staging 
examination and referral for treatment; participants consented specifically prior to review of medical records. Research assistants were present in the clinic and were recruiting about $50 \%$ of the time during the study period.

Follow-up contact information was obtained. A telephone survey regarding treatment initiation was administered a minimum of 4 weeks and up to 3 months after the initial interview. Those who had not been able to access treatment were offered a follow-up visit and/or directions for follow-up in radiation oncology, medical oncology or palliative care, as appropriate.

Survey data were captured on tablets using Open Data Kit (ODK) software (https://opendatakit.org). ${ }^{28}$ Data were uploaded daily and aggregated on a secure server.

\section{Patient and public involvement}

We developed the recruitment and survey procedures in collaboration with UCI and MNRH clinic staff. The data collection team (nurses with extensive experience in the field) provided feedback on the survey instrument. The survey was pilot-tested on 20 patients in the clinics who were otherwise eligible, gave informed consent and were partially compensated for travel expenses, in order to ensure that the questions were understandable and that the response options were relevant and comprehensive. The survey was revised to incorporate the pretest feedback from patients and research assistants. Findings have not been disseminated, per se, but as mentioned previously this analysis is part of a larger study examining patterns of delay in accessing treatment in order to inform the design of an intervention to minimise delay and improve access to care.

\section{Ethics statement}

The informed consent document and the survey were translated into Luganda; English is the official language of instruction at Makerere University College of Medical Sciences. Informed consent was obtained and documented from all participating survey responders. Participants were assigned a unique identification number for data collected over the course of the study to de-identify participants and maintain confidentiality. Encrypted software and secure databases were used. No participant-identifying information will be disclosed in any publication.

\section{Sample size}

As this was a largely descriptive study, our power considerations focused on acceptably precise estimates of proportions. To calculate our sample size, we assumed that if $20 \%$ of women with cervical cancer in Uganda were diagnosed with early-stage cervical cancer (I-IIA), a total of 265 women would allow us to experimentally determine this proportion within 5\% (confidence level 95\%). We thus aimed to include at least 265 patients using consecutive sampling techniques. Hysterectomy would be a theoretical treatment option for those with stage IA-IIA, although we expected not all would be recommended for primary surgery. We estimated the proportion of surgical candidates, our outcome of interest, in the total sample would likely be $10 \%-15 \%$; thus, a sample size of 265 would allow us to report the proportion of surgical candidates within 3-4 percentage points (confidence level 95\%).

\section{Outcomes}

Our primary outcome variable, surgical candidacy, was defined as a recommendation for hysterectomy (simple or radical) as primary treatment. While any patient with 'early-stage' disease (IA-IIA) may have been considered for hysterectomy, we used the physician's actual recommendation, after clinical assessment, to define 'candidacy'. Any 'late-stage' (IIB-IVB) patients recommended for surgery were dropped, as this would likely be a recommendation for palliative surgery, not definitive primary therapy with curative intent. Treatment initiation, our other outcome variable, was defined by a participant selfreporting starting any treatment, including hospice.

\section{Statistical analyses}

To test for associations between categorical outcome variables (surgical candidacy and treatment initiation) and categorical explanatory variables, we performed $\chi^{2}$ tests and Fisher's exact tests. For continuous explanatory predictors, we used two independent sample t-tests to compare sample means by outcome. We used logistic regression to explore the association of demographic and reproductive health variables with these outcomes, adjusting to limit bias from confounding. Factors theorised to be associated with the outcomes or those found to be significantly associated in bivariate analysis were considered for multivariate analysis. Because only those with early-stage disease could be surgical candidates (see Outcomes section; surgical candidates are a subset of early-stage disease), the variables were not independent; thus, we could not adjust analysis of surgical candidacy for stage. Listwise deletion was used to remove observations missing outcome or predictor variables. Sensitivity analyses were performed to examine outcomes in specific subgroups, specifically by stage and availability of radiation. All data were analysed using Stata V.14.0. P values less than 0.05 were considered statistically significant.

\section{RESULTS}

From April 2017 through September 2018, 332 women were screened and ultimately 268 participated (see figure 1). Among the participants, 233 (87\%) initially presented with symptoms. Most women $(n=204,76 \%)$ were diagnosed at an advanced stage (IIB-IVB), and these women, compared with those with early-stage disease (IA1-IIA) $(\mathrm{n}=64,24 \%)$, were more likely to present with symptoms including bleeding, discharge, pain or fatigue (OR 2.44, 95\% CI 1.16 to 5.14) rather than through routine screening. About half of those with early-stage disease were recommended to undergo hysterectomy as 


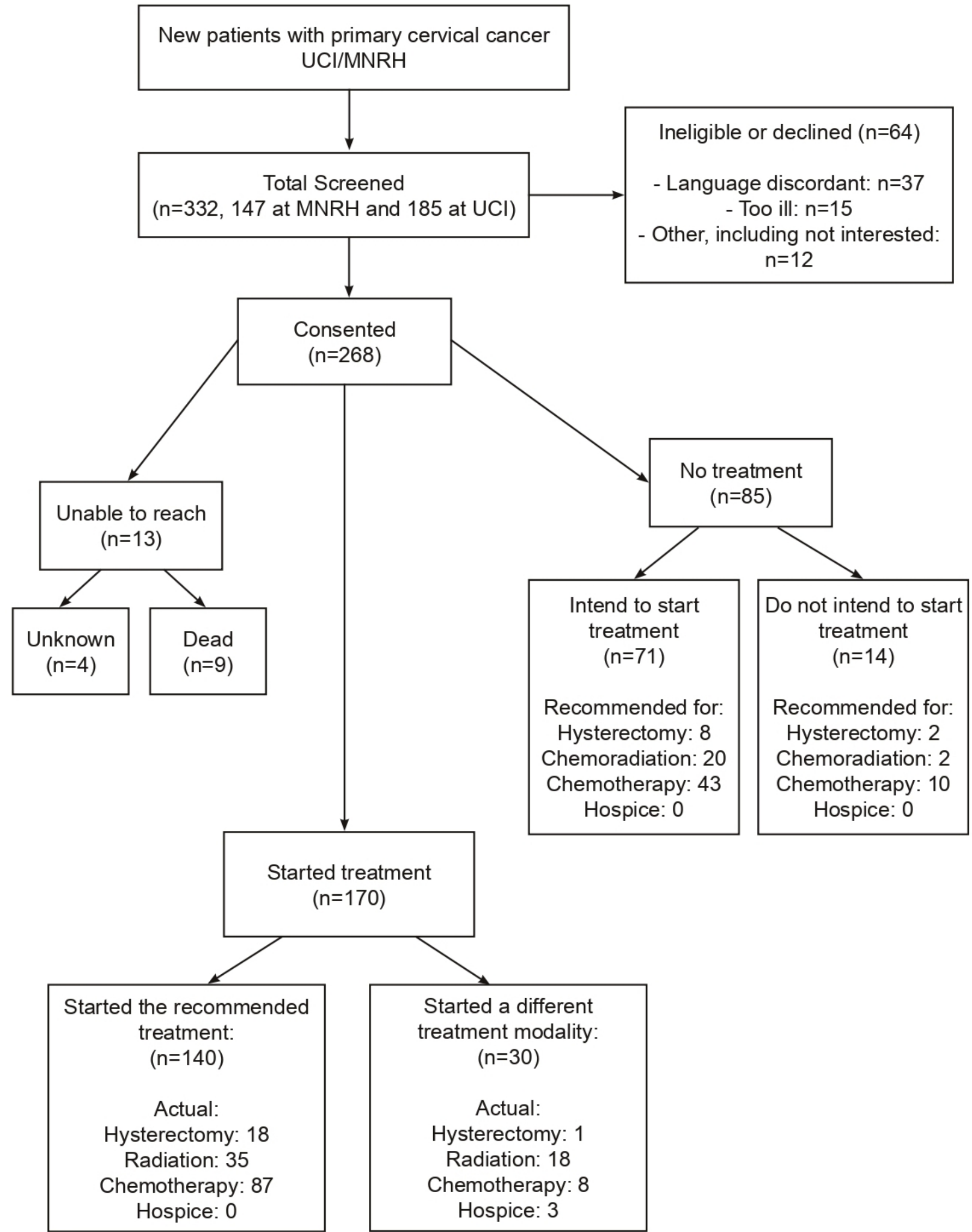

Figure 1 Study flow diagram. This flow chart depicts the treatment uptake patterns of study participants according to whether or not they had started treatment, intention to start and whether or not the treatment initiated was concordant with treatment recommendations. MNRH, Mulago National Referral Hospital; UCI, Uganda Cancer Institute. 
Table 1 Characteristics of women diagnosed with cervical cancer, by surgical candidacy

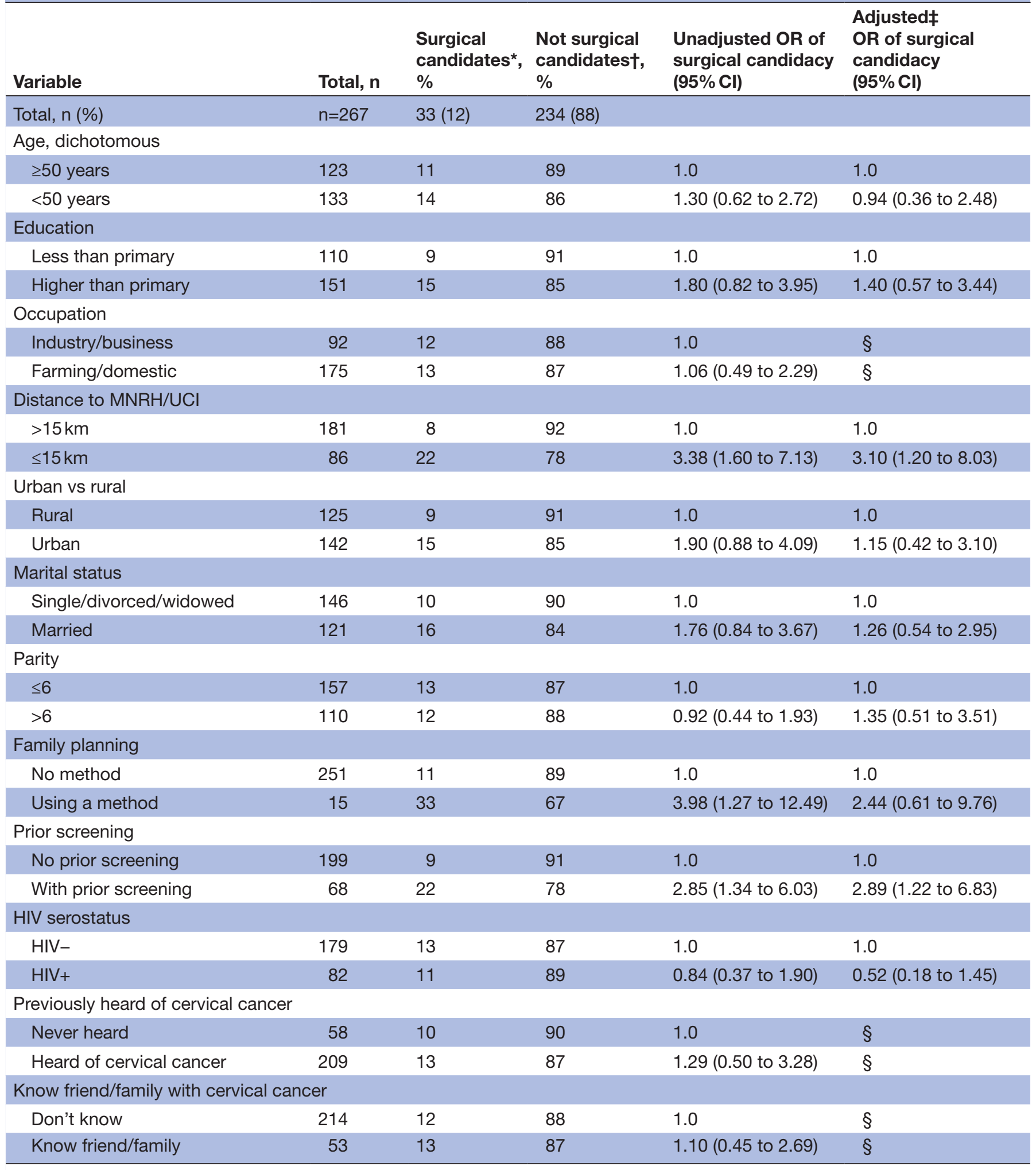

*As recommended by specialists at $\mathrm{UCI} / \mathrm{MNRH}$ for hysterectomy (simple or radical); eliminating one woman with locally advanced disease who reported a recommendation for surgery.

†Recommended for primary treatment with chemoradiation, chemotherapy or hospice.

$\ddagger$ Adjusted for age, education, distance, urban residence, marital status, parity, family planning use, prior screening and HIV serostatus.

$\S$ Not included in the multivariate model.

$\mathrm{MNRH}$, Mulago National Referral Hospital; UCI, Uganda Cancer Institute. 
primary treatment $(\mathrm{n}=33,52 \%$ of those with early-stage disease, $12 \%$ of total).

In unadjusted analysis, living in or within $15 \mathrm{~km}$ of Kampala (OR 3.38, 95\% CI 1.60 to 7.13), current use of modern family planning (OR 3.98, 95\% CI 1.26 to 12.49 ) and prior screening (OR 2.85, 95\% CI 1.34 to 6.03) were associated with a recommendation for surgery. In multivariate analysis, living within $15 \mathrm{~km}$ of Kampala (OR $3.10,95 \%$ CI 1.20 to 8.03 ) and prior screening (OR 2.89, $95 \%$ CI 1.22 to 6.83 ) remained significantly associated with a recommendation for surgery as primary treatment (see table 1).

Recommended treatments varied by stage, as expected. We hypothesised that recommendations may also vary by availability of radiation therapy (see table 2 ). Almost two-thirds of the participants $(\mathrm{n}=167,62 \%)$ presented for care at a time when no incountry external beam radiation was available, compared with 101 participants $(38 \%)$ who received treatment recommendations after the new machine was commissioned.

Most women with stage IA1-IB1 disease $(n=35)$ were recommended for surgery regardless of availability of incountry radiation $(n=32,92 \%)$. Among women with stage IB2-IIA disease $(n=29)$, only one was recommended to undergo hysterectomy and this was when the radiation machine was broken down. Chemotherapy (likely neoadjuvant) was recommended as the primary treatment to most of those with IB2-IIA disease, regardless of availability of radiation (19 of $29,66 \%$ overall; $75 \%$ when radiation machine was broken down vs $54 \%$ when machine was working; OR 2.57, 95\% CI 0.53 to 12.38 ).

However, for women with locally advanced disease (stage IIB-IIIB), absence of incountry radiation was associated with significantly higher odds of a recommendation for chemotherapy (OR $15.43,95 \%$ CI 7.16 to 33.25 ) and lower odds of a recommendation for chemoradiation (OR $0.06,95 \%$ CI 0.03 to 0.13 ). Few women $(\mathrm{n}=14)$ had stage IV disease and none of these women was recommended for radiation when the machine was down. Only two participants, both stage IV, were recommended to start hospice.

We obtained follow-up information for 264 of the 268 participants. Nine women died before starting treatment. Among the 255 living women reached for phone interview, $170(67 \%)$ had initiated some treatment. No demographic or health or cancer-related variables (including treatment recommendations and radiation availability) were associated with treatment initiation in univariate analysis (see table 3). Given lack of association, multivariate analysis was not performed.

Of the 33 women recommended to undergo hysterectomy, $55 \%$ had surgery, another $15 \%$ started other treatment modalities and the remaining $30 \%$ had not started any treatment (see figure 2; of note, a woman at stage IB1 who was initially recommended for chemotherapy ultimately had hysterectomy). Among the 170 women who started treatment, most $(\mathrm{n}=140,82 \%)$ started the treatment modality that was recommended. Only young age ( $<50$ years) was associated with higher odds of starting the recommended treatment (OR 1.84, 95\% CI 1.11 to 3.07); there was no association with any other demographic or health or cancer-related factors (including stage, treatment recommendations and radiation availability).

Of the 85 participants who had not started treatment, $71(84 \%)$ planned to start. The most commonly reported reasons for delay were financial constraints, including lack of funds to pay for travel and the nominal fees associated with radiation, surgery and diagnostic tests $(69 \%)$ and long wait times $(30 \%)$. For the subset of these women recommended for hysterectomy $(n=8)$, the most common reasons for delay were the same: financial constraints $(50 \%)$ and perceived long wait $(37.5 \%)$. For the 14 participants not planning to start any treatment or hospice, the most common reasons were pursuing

Table 2 Treatment recommendations by stage and availability of radiation

\begin{tabular}{|c|c|c|c|c|c|}
\hline Stage at presentation & Radiation availability & $\begin{array}{l}\text { Surgery, } \\
\text { n (\%) }\end{array}$ & $\begin{array}{l}\text { Chemoradiation*, } \\
\text { n (\%) }\end{array}$ & $\begin{array}{l}\text { Chemotherapyt, } \\
\text { n (\%) }\end{array}$ & $\begin{array}{l}\text { Hospice, } \\
\text { n (\%) }\end{array}$ \\
\hline $\begin{array}{l}|A 1-| B 1 \\
n=35\end{array}$ & No RT available $(n=23)$ & $21(92)$ & $1(4)$ & $1(4)$ & 0 \\
\hline \multirow{2}{*}{$\begin{array}{l}\text { IB2-IIA } \\
n=29\end{array}$} & No RT available $(n=16)$ & $1(6)$ & $3(19)$ & $12(75)$ & 0 \\
\hline & RT available $(n=13)$ & 0 & $6(46)$ & $7(54)$ & 0 \\
\hline $\begin{array}{l}\text { IIB-IIIB } \\
n=190\end{array}$ & RT available $(n=71)$ & 0 & $45(63)$ & $26(37)$ & 0 \\
\hline \multirow{2}{*}{$\begin{array}{l}\text { IVA-IVB } \\
n=14\end{array}$} & No RT available $(n=9)$ & 0 & 0 & $8(89)$ & $1(11)$ \\
\hline & RT available $(n=5)$ & 0 & $2(40)$ & $2(40)$ & $1(20)$ \\
\hline
\end{tabular}

\footnotetext{
*All patients recommended for external beam radiation were also recommended to have concurrent weekly chemosensitisation, typically with cisplatin $40 \mathrm{mg}$ per square metre concurrent with external beam pelvic radiation, typically 4500 cGy in 25 fractions. †Chemotherapy recommended as primary modality, either in neoadjuvant setting (stage IB1-IIIB) or for distant metastases (stage IV). Doublet therapy with cisplatin $75 \mathrm{mg}$ per square metre and paclitaxel $135-175 \mathrm{mg}$ per square metre every 3 weeks was typically the standard recommended regimen.

$\mathrm{RT}$, radiotherapy.
} 
Table 3 Characteristics of participants by whether or not they had started any treatment

Unadjusted OR of treatment initiation

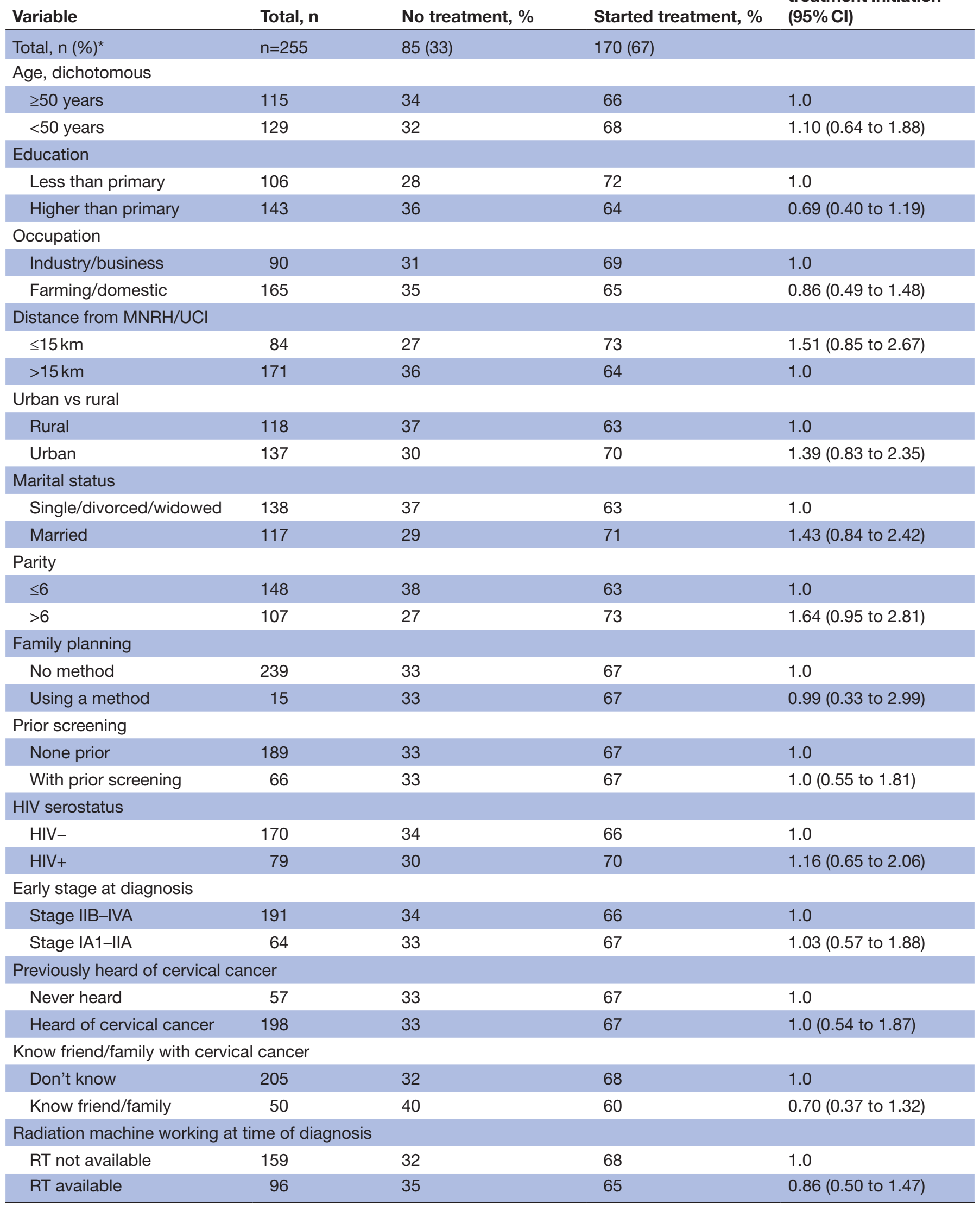

Continued 
alternative or traditional methods $(57 \%)$ and/or financial constraints $(50 \%)$. Of these 14 participants, 2 had been recommended for hysterectomy: one cited 'a long queue' and the other distrust of treatment recommendation as the reason for not pursuing treatment.

\section{DISCUSSION}

In this cohort of women with cervical cancer presenting at referral centres in Kampala, Uganda, three-quarters presented at an advanced stage (IIB-IVB) and only $12 \%$ were recommended to undergo hysterectomy. Overall, treatment recommendations were in line with the ASCO Resource-Stratified Clinical Practice Guideline. ${ }^{19}$ The significance of the availability of radiation in Uganda on treatment recommendations seemed to vary based on stage, although the study was underpowered to assess these effects by subgroup.

This study adds to the epidemiology of cervical cancer in the region and is the first study in the region to report on the proportion of patients with newly diagnosed cervical cancer recommended for hysterectomy. The proportion of participants presenting at late stage (IIB-IVB), 76\%, is similar to 20-year-old estimates from the Kampala Cancer Registry, ${ }^{6}$ as well as to more recent regional estimates from Northern Uganda, ${ }^{5}$ Ghana ${ }^{29}$ and Rwanda. ${ }^{30}$ Given the lack of national screening and nascent human papillomavirus vaccination efforts, it is unsurprising that the proportion of late-stage disease is unchanged over the last two decades in Uganda.

As expected, women with early-stage disease were more likely to be assessed as surgical candidates. Although radical hysterectomy is a theoretical consideration for stage IB2-IIA disease, chemoradiation, when available, is preferred. We expected more of these women to be recommended for primary surgery when the radiation machine was out of commission. In this subset (stage IB2-IIA), we did not find an increased recommendation for radical hysterectomy during the time without incountry radiation, although the study was underpowered to assess this impact. Although large tumours $(>4 \mathrm{~cm})$ are not necessarily associated with increased complications among experienced surgeons, ${ }^{23} 31$ newly fellowship-trained providers with limited resources including lack of equipment and cross-matched blood may not be eager to offer radical hysterectomy to stage IB2-IIA patients.

Surprisingly, the majority of women with stage IB2-IIA disease were recommended for chemotherapy (likely neoadjuvant), regardless of the availability of radiation. As chemoradiation is the preferred modality, we would have expected more of these women to be referred for radiation when the new machine was operational. But availability is not synonymous with accessibility; prior to discontinuation, the old machine, despite running 20 hours per day, was meeting just $2.6 \%$ of the nation's indicated radiation treatments. ${ }^{14}$ Alternatively, it is possible a perception of success resulted in a continuation of the practice pattern of neoadjuvant chemotherapy. These data are forthcoming.

Two-thirds of women in the current study had initiated treatment; neither stage, recommended treatment nor radiation availability predicted treatment initiation. Disappointingly, only $55 \%$ of the women recommended for curative hysterectomy were able to undergo surgery. Women unable to access hysterectomy reported the same challenges as women recommended for chemoradiation or chemotherapy.

There are comparable regional data on radiation initiation. A survey from rural Rwanda reported that $80 \%$ of the patients with cervical cancer at the Butaro Cancer Center of Excellence (BCCOE) who were referred to UCI for chemoradiation (there is no available radiation in Rwanda) were able to access treatment. ${ }^{30}$ This high proportion of treatment initiation contrasts our findings that women were generally unable to access outof-country radiation. As expected, in our study, women with locally advanced disease (stage IIB-IIIB) were less likely to be recommended for standard-of-care chemoradiation when there was no available incountry radiation. For those recommended to undergo chemoradiation in Kenya, when the radiation machine in Uganda was down $(n=15)$, only two of them reported actually starting chemoradiation, whereas when the Kampala-based machine was working two-thirds of women were able to access this treatment. 


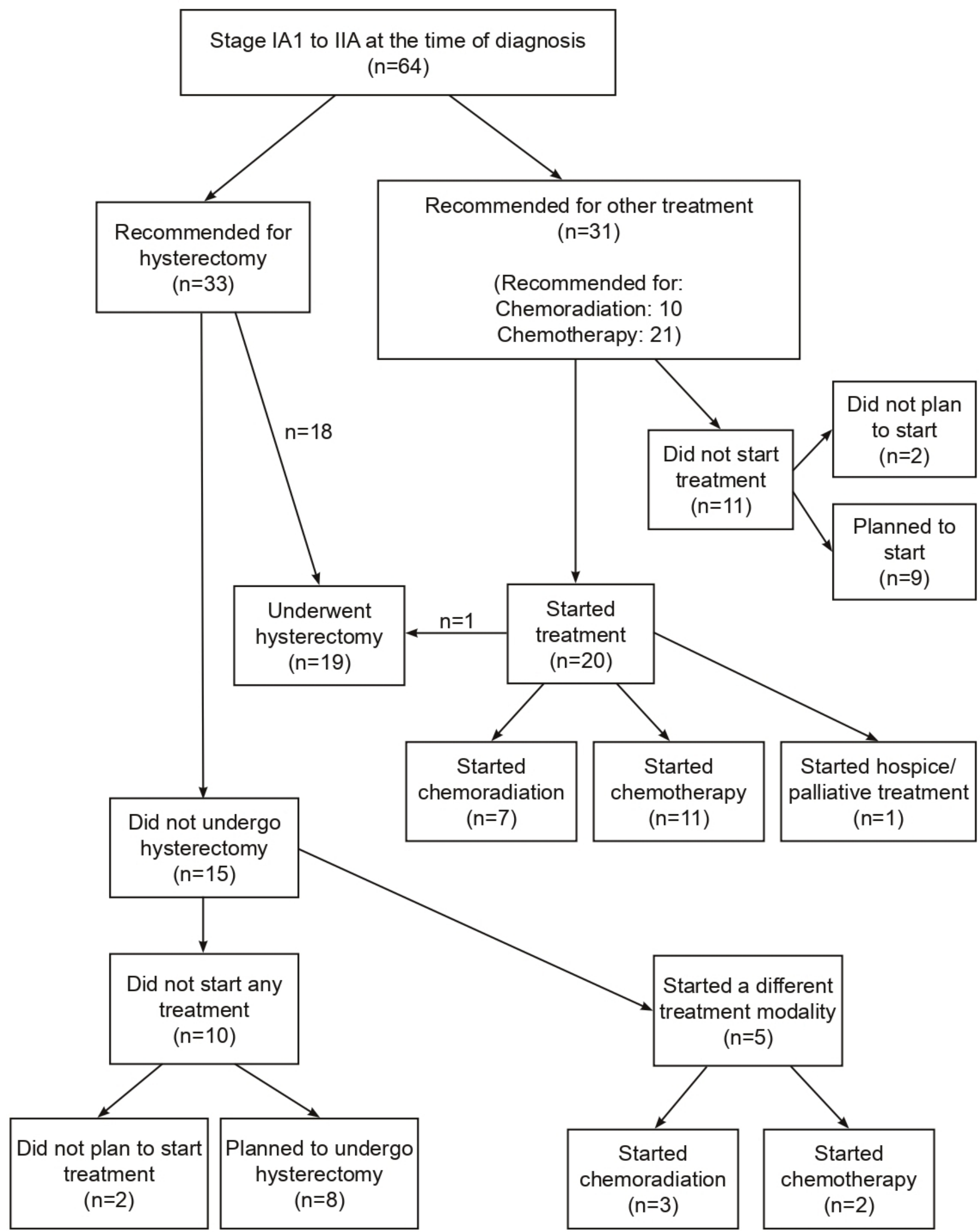

Figure 2 Treatment recommendations and uptake of women with early-stage disease (stage IA1-IIA at time of diagnosis), especially those recommended for hysterectomy versus chemoradiation or chemotherapy. 
The most commonly cited reason for failing to access treatment, including curative hysterectomy, financial hardship, is difficult for the underfunded public health sector to modify. Nearly half of the country's healthcare financing continues to come from out-of-pocket expenses. ${ }^{32}$ Clinical consultations are free of charge and there are nominal fees associated with radiation, surgery, labs and imaging. While nominal, these fees are still out of reach for the majority of the country's population with an average monthly income of 300000 Ugandan shillings (about US\$80). Additionally, most of the population lives in rural areas outside the capital, where no cancer care is available, necessitating travel and lodging, with associated expense.

The second most cited reason, long wait times, implies an unmet need for treatment, including radical hysterectomies. As the burden of cancer shifts to LMICs, a recalibrated response from the international community is necessary to substantially increase funding for capacity building and training opportunities for local clinicians, as well as for sufficient equipment and supplies to enable oncological surgery, radiation and chemotherapy. ${ }^{33} \mathrm{UCI}$ and MNRH, in partnership with international mentors and funding, are to be commended for creating the country's first gynaecological oncology fellowship to locally train subspecialists.

Generally speaking, most participants had previously heard of cervical cancer, similar to reports from Northern Uganda, ${ }^{34}$ but neither knowledge nor knowing someone with cervical cancer was associated with surgical candidacy or successful treatment initiation. Thus, education efforts designed to increase awareness of cervical cancer would not likely increase the proportion of women diagnosed at an early stage in the absence of screening and referral services.

This study highlights the prevalence of late presentation to cervical cancer care and suggests that distance from treatment facilities and general poor access to care (as evidenced by lack of prior screening) affect women's eligibility for curative surgery. These risks are difficult to modify, but suggest that either disseminating specialty cancer care beyond large urban centres, as the Rwandan Ministry of Health did with the BCCOE, or strengthening the referral system between rural health centres and the referral hospitals may improve timeliness to care.

While this study provides a snapshot of cervical cancer epidemiology, treatment recommendations and uptake, the population is a convenience sample of women who were able to access care at referral centres, limiting generalisability. The study population likely represents a conservative estimate of those at a late stage at time of presentation and likely overestimates the proportion of women nationally who may be surgical candidates at the time of diagnosis. The myriad challenges associated with providing cancer treatment, including lack of available operating theatres, surgical equipment, anaesthesiologists and trained surgeons for early-stage disease, as well as lack of radiation, chemotherapy and radiation and medical oncologists for late-stage disease, underscore the need to expand prevention and screening opportunities in Uganda.

Further research is needed to assess when and why women experience delay in accessing care. Additionally, hospice and palliative care, although widely available in Uganda and subsidised, seemed to be poorly used. Given the substantial proportion of women presenting at late stage, the nine women who died before accessing the recommended treatment as well as the significant proportion of women who were not able to access any treatment, hospice is likely an appropriate treatment strategy for many women. Future research is needed to understand barriers specific to uptake of palliative care and hospice.

While decreasing the incidence of cervical cancer will only be possible by expanding vaccination and screening opportunities, efforts to improve earlier detection and diagnosis, expand accessibility of surgery, radiation and chemotherapy, and integrate palliative care into standard treatment are essential to decrease mortality, morbidity and suffering among women with cervical cancer.

\section{Author affiliations}

${ }^{1}$ Obstetrics, Gynecology and Reproductive Sciences, University of California San Francisco, San Francisco, California, USA

${ }^{2}$ Obstetrics and Gynaecology, Makerere University College of Health Sciences, Kampala, Uganda

${ }^{3}$ Obstetrics and Gynaecology, Mulago National Referral Hospital, Kampala, Uganda

${ }^{4}$ Uganda Cancer Institute, Kampala, Uganda

${ }^{5}$ Duke Global Health Institute, Durham, North Carolina, USA

${ }^{6}$ Obstetrics and Gynecology, Duke University School of Medicine, Durham, North Carolina, USA

Acknowledgements The authors would like to acknowledge the data collection and patient advisory team: Najjemba Irene, Najjemba Beatrice, Nakadu Laurant and Nankya Esther. Lindsay Swanson is also acknowledged for figure design.

Contributors Conception and design: MS, MN, MJH, LC, SU. Administrative support: MN, JN, CN. Provision of study materials or patients: MN, JN, CN. Collection and assembly of data: MS, MN. Data analysis and interpretation: MS, MJH. Manuscript writing: MS, MJH. Final approval of manuscript: all. Accountable for all aspects of this work: all.

Funding This work was supported by NIH Research Training Grant \#D43 TW009343 funded by the Fogarty International Center, as well as the University of California Global Health Institute.

Competing interests None declared.

Patient consent for publication Not required.

Ethics approval This study was reviewed and approved by several institutional review boards: University of California San Francisco Human Research Protection Program Institutional Review Board (IRB \#16-21306), Mulago Research and Ethics Committee (MREC \#1058), Uganda Cancer Institute Research and Ethics Review Committee, and Uganda National Council for Science and Technology (reference \#HS29Es).

Provenance and peer review Not commissioned; externally peer reviewed.

Data availability statement De-identified data are available upon reasonable email request from the corresponding author.

Open access This is an open access article distributed in accordance with the Creative Commons Attribution Non Commercial (CC BY-NC 4.0) license, which permits others to distribute, remix, adapt, build upon this work noncommercially, and license their derivative works on different terms, provided the original work is properly cited, appropriate credit is given, any changes made indicated, and the use is non-commercial. See: http://creativecommons.org/ licenses/by-nc/4.0/. 
ORCID iD

Megan Swanson http://orcid.org/0000-0003-0881-7923

\section{REFERENCES}

1 Bray F, Ferlay J, Soerjomataram I, et al. Global cancer statistics 2018: GLOBOCAN estimates of incidence and mortality worldwide for 36 cancers in 185 countries. CA Cancer J Clin 2018;68:394-424.

2 Bruni L B-RL, Albero G, Aldea M, et al. Human papillomavirus and related diseases in Uganda. summary report 2016- 02-26: ICO information centre on HPV and cancer (HPV information centre, 2016.

3 Wabinga HR, Nambooze S, Amulen PM, et al. Trends in the incidence of cancer in Kampala, Uganda 1991-2010. Int J Cancer 2014;135:432-9.

4 Ndejjo R, Mukama T, Musabyimana A, et al. Uptake of cervical cancer screening and associated factors among women in rural Uganda: a cross sectional study. PLoS One 2016;11:e0149696.

5 Mwaka AD, Garimoi CO, Were EM, et al. Social, demographic and healthcare factors associated with stage at diagnosis of cervical cancer: cross-sectional study in a tertiary hospital in northern Uganda. BMJ Open 2016;6:e007690.

6 Wabinga $\mathrm{H}$, Ramanakumar AV, Banura $\mathrm{C}$, et al. Survival of cervix cancer patients in Kampala, Uganda: 1995-1997. Br J Cancer 2003;89:65-9.

7 Gondos A, Brenner $\mathrm{H}$, Wabinga $\mathrm{H}$, et al. Cancer survival in Kampala, Uganda. Br J Cancer 2005;92:1808-12.

8 Sullivan R, Alatise OI, Anderson BO, et al. Global cancer surgery: delivering safe, affordable, and timely cancer surgery. Lancet Oncol 2015;16:1193-224.

9 Johnston C, $\mathrm{Ng} \mathrm{JS}$, Manchanda R, et al. Variations in gynecologic oncology training in low (LIC) and middle income (MIC) countries (LMICs): common efforts and challenges. Gynecol Oncol Rep 2017;20:9-14.

10 Meara JG, Leather AJM, Hagander L, et al. Global surgery 2030: evidence and solutions for achieving health, welfare, and economic development. Int J Obstet Anesth 2016;25:75-8.

11 Dubowitz G, Detlefs S, McQueen KAK. Global anesthesia workforce crisis: a preliminary survey revealing shortages contributing to undesirable outcomes and unsafe practices. World J Surg 2010;34:438-44

12 Epiu I, Tindimwebwa JVB, Mijumbi C, et al. Challenges of anesthesia in low- and middle-income countries: a cross-sectional survey of access to safe obstetric anesthesia in East Africa. Anesth Analg 2017:124:290-9.

13 Lund TC, Hume H, Allain JP, et al. The blood supply in sub-Saharan Africa: needs, challenges, and solutions. Transfus Apher Sci 2013;49:416-21.

14 Abdel-Wahab M, Bourque J-M, Pynda Y, et al. Status of radiotherapy resources in Africa: an international atomic energy agency analysis. Lancet Oncol 2013;14:e168-75.

15 Datta NR, Samiei M, Bodis S. Radiation therapy infrastructure and human resources in low- and middle-income countries: present status and projections for 2020. Int J Radiat Oncol Biol Phys 2014;89:448-57.

16 Mugerwa Y. Govt to airlift 400 cancer patients to Kenya. Daily Monitor, 2016.
17 Nelson KG. Waiting to die: Uganda's untreated cancer patients. Aljazeera, 2017.

18 Barr R, Robertson J. Access to cytotoxic medicines by children with cancer: a focus on low and middle income countries. Pediatr Blood Cancer 2016;63:287-91.

19 Chuang LT, Temin S, Camacho R, et al. Management and care of women with invasive cervical cancer: American Society of clinical oncology Resource-Stratified clinical practice guideline. J Glob Oncol 2016;2:311-40.

20 Benedetti-Panici P, Greggi S, Colombo A, et al. Neoadjuvant chemotherapy and radical surgery versus exclusive radiotherapy in locally advanced squamous cell cervical cancer: results from the Italian multicenter randomized study. J Clin Oncol 2002;20:179-88.

21 Chang TC, Lai $\mathrm{CH}$, Hong JH, et al. Randomized trial of neoadjuvant cisplatin, vincristine, bleomycin, and radical hysterectomy versus radiation therapy for bulky stage $\mathrm{Ib}$ and IIA cervical cancer. J Clin Oncol 2000;18:1740-7.

22 Kokka F, Bryant A, Brockbank E, et al. Hysterectomy with radiotherapy or chemotherapy or both for women with locally advanced cervical cancer. Cochrane Database Syst Rev 2015;20:Cd010260.

23 Landoni F, Maneo A, Colombo A, et al. Randomised study of radical surgery versus radiotherapy for stage IB-IIA cervical cancer. Lancet 1997;350:535-40.

24 Unger-Saldaña K, Peláez-Ballestas I, Infante-Castañeda C. Development and validation of a questionnaire to assess delay in treatment for breast cancer. BMC Cancer 2012;12:626.

25 Scott SE, Walter FM, Webster A, et al. The model of pathways to treatment: conceptualization and integration with existing theory. $\mathrm{Br} \mathrm{J}$ Health Psychol 2013;18:45-65.

26 Walter F, Webster A, Scott S, et al. The Andersen model of total patient delay: a systematic review of its application in cancer diagnosis. J Health Serv Res Policy 2012;17:110-8.

27 Andersen BL, Cacioppo JT. Delay in seeking a cancer diagnosis: delay stages and psychophysiological comparison processes. $\mathrm{Br} J$ Soc Psychol 1995;34 Pt 1:33-52.

28 Hartung C, Lerer A, Anokwa Y, et al. Open data kit: tools to build information services for developing regions. In: Proceedings of the 4th ACM/IEEE International Conference on information and communication technologies and development. London, United Kingdom: ACM, 2010: 1-12.

29 Dunyo P, Effah K, Udofia EA. Factors associated with late presentation of cervical cancer cases at a district Hospital: a retrospective study. BMC Public Health 2018;18:1156.

30 Park PH, Davey S, Fehr AE, et al. Patient characteristics, early outcomes, and implementation lessons of cervical cancer treatment services in rural Rwanda. J Glob Oncol 2018;4:1-11.

31 Machida H, Matsuo K, Furusawa A, et al. Profile of treatment-related complications in women with clinical stage IB-IIB cervical cancer: a nationwide cohort study in Japan. PLoS One 2019;14:e0210125.

32 Orem JN, Zikusooka CM. Health financing reform in Uganda: how equitable is the proposed national health insurance scheme? Int $J$ Equity Health 2010;9:23.

33 Swanson M, Ueda S, Chen L-M, et al. Evidence-Based improvisation: facing the challenges of cervical cancer care in Uganda. Gynecol Oncol Rep 2018;24:30-5.

34 Mwaka AD, Orach CG, Were EM, et al. Awareness of cervical cancer risk factors and symptoms: cross-sectional community survey in post-conflict Northern Uganda. Health Expect 2016;19:854-67. 